\title{
Irradiation of successive pulsed and continuous-wave lasers on a transparent polyethylene film
}

\author{
Changqing Song, ${ }^{\text {a,b }}$ Kai Han,, a,b, $*$ Xiao Li, ${ }^{\text {a,b }}$ Wenda Cui, ${ }^{\text {a,b }}$ \\ and Xiaojun $\mathrm{Xu}^{\mathrm{a}, \mathrm{c}}$ \\ ${ }^{a}$ National University of Defense Technology, College of Advanced Interdisciplinary Studies, \\ Changsha, China \\ ${ }^{b}$ National University of Defense Technology, State Key Laboratory of Pulsed Power Laser \\ Technology, Changsha, China \\ ${ }^{\mathrm{c}}$ National University of Defense Technology, Hunan Provincial Key Laboratory of High Energy \\ Laser Technology, Changsha, China
}

\begin{abstract}
It is difficult to cut through a transparent material such as polyethylene (PE) film with a continuous-wave $(\mathrm{CW})$ laser of the near-infrared wavelength, because of the low absorption of laser energy. The plasma induced by a pulsed laser, however, can damage the surface of a film, which can change the transparency of the material. In this study, a transparent PE film with a thickness of $0.6 \mathrm{~mm}$ was irradiated by various combinations of focused laser pulses and a $\mathrm{CW}$ laser. The test conditions and the processes were recorded visually by a Schlieren optics system on the side surface of the film and by a high-speed camera on the front surface. From the results, it can be concluded that, though it is difficult to cut through a transparent material such as PE film with only a pulsed or CW laser alone, once the transparency of the surface has been modified by multiple focused laser pulses, the PE film can be cut through easily by a CW laser with a nearinfrared wavelength. (C) The Authors. Published by SPIE under a Creative Commons Attribution 4.0 Unported License. Distribution or reproduction of this work in whole or in part requires full attribution of the original publication, including its DOI. [DOI: 10.1117/1.OE.59.12.125103]
\end{abstract}

Keywords: irradiation; pulsed laser; continuous-wave laser; polyethylene film; cut through time.

Paper 20200995 received Aug. 20, 2020; accepted for publication Nov. 20, 2020; published online Dec. 4, 2020.

\section{Introduction}

Pulsed and continuous-wave (CW) lasers with near-infrared wavelength are widely used to cut materials in manufacturing processes. ${ }^{1}$ The cutting process is determined by the interaction between the laser beam and the sample, which leads to ablation and consequently the formation of laser-ablated craters on the sample surface. When the sample is a transparent material such as polyethylene (PE) film with smooth surfaces, the cutting effect is not good. Because the PE film has very low energy absorption at the wavelength of $1064 \mathrm{~nm}$, most of the laser energy is reflected or transmitted; the threshold for the $\mathrm{CW}$ laser of $1064 \mathrm{~nm}$ to cut through the film is evaluated to be higher than $600 \mathrm{~W} / \mathrm{cm}^{2}$, which is too high.

Two methods are now used to cut the PE film. One method is changing the wavelength of $\mathrm{CW}$ laser to another wavelength with a higher absorption, such as mid-infrared wavelength, but another laser source will be needed. Another method is improving the power of the CW laser to a higher level to be able to cut through the PE film, but this method is more costly. Also, when a higher-power laser of near-infrared wavelength is used to cut through the PE film, the high reflected and transmitted power of the laser may cause danger. If we want to cut the PE film in the same manner as other untransparent materials with the near-infrared laser of $1064 \mathrm{~nm}$, and do not want the required power of the CW laser source be high, other method needs to be sought. This paper aims to find another method to decrease the threshold for the near-infrared CW laser of $1064 \mathrm{~nm}$ to cut through the transparent PE film. Once the threshold is greatly decreased, a

*Address all correspondence to Kai Han, hankai0071@nudt.edu.cn 
near-infrared CW laser with low power can be used to cut the transparent PE materials, and potentially may be used to burn the PE materials when needed in the future, such as for cleaning up transparent plastic bags made of PE film hanging on high-voltage wires.

Chen et al. ${ }^{2}$ showed that plasma induced by a pulsed laser can damage the surface of a $\mathrm{Ta}_{2} \mathrm{O}_{5}$ film, which can significantly reduce the transmittance of the film. Once the transparency of the film is changed by a pulsed laser, it might be easier for the same CW laser to cut through the film. This study focuses on the irradiation of a PE film by successive pulsed and CW laser sources, with the objective of decreasing the threshold for a CW laser to cut through the film.

There has been inadequate research into the damage to transparent films by successive pulsed and $\mathrm{CW}$ laser beams, and particularly to transparent PE films. Because this damage is mainly caused by the induced plasma, the characteristics of laser-induced plasmas must be understood. Chylek et al. ${ }^{3}$ and Phuoc ${ }^{4}$ measured the laser-induced breakdown threshold for air, which is the minimum power intensity for the laser to induce air breakdown. With the development of highspeed imaging instrumentation, the dynamic process of laser-induced plasma was photographed. Sinibaldi et al. ${ }^{5}$ analyzed the plasma generated by a laser in liquid, and the structure of the breakdown plasma and the ensuing bubble dynamics in the liquid were recorded. Brieschenk et al. ${ }^{6,7}$ recorded pictures of the early evolution of a laser-induced plasma explosion in air using highspeed time-resolved Schlieren images. These images showed that the plasma was initiated in the focal waist of the laser beam, and formed an elongated spheroid oriented along the same major axis as the laser beam. Two counter-rotating toroidal vortices were formed in the posterior and anterior regions of the kernel with two gaseous jets, all flowing to the center of the kernel. The jet in the posterior region prevailed in the collision, and a gaseous jet formed propagating toward the direction of the laser source.

Although there have been many studies on laser-induced plasma, the process was unclear when there was a transparent film placed near the focal waist of the pulsed laser beam, especially when the laser energy was less than the threshold for laser-induced breakdown in air. Ding et al. ${ }^{8}$ analyzed the combined irradiations of pulsed and $\mathrm{CW}$ laser beams, where the ablated materials were silicon and stainless steel. Zhou et al. ${ }^{9}$ studied the $\mathrm{TiO}_{2} / \mathrm{SiO}_{2}$ dielectric film damage induced by the combined irradiations from pulsed and $\mathrm{CW}$ lasers, which showed the advantages of the combined lasers. Sohn et al. ${ }^{10}$ micropatterned a transparent polypropylene film with the thickness of $30 \mu \mathrm{m}$, and the results demonstrated that the femtosecond pulsed laser was an efficient tool.

In this study, a damaging experiment of a pulsed laser on a PE film was conducted, the film was placed at the focal waist of the laser beam. After irradiation by different numbers of laser pulses, a CW laser with the same wavelength of $1064 \mathrm{~nm}$ will be used to burn the damaged PE film.

\section{Experimental Techniques}

The PE film used in the experiments was ordinary transparent PE with smooth front and back surfaces. The PE film was a commercially used film bought from an online shop. A specially designed support was used to fix the position of the PE film in the test, as shown in Fig. 1. The thickness of the film was $0.6 \mathrm{~mm}$.

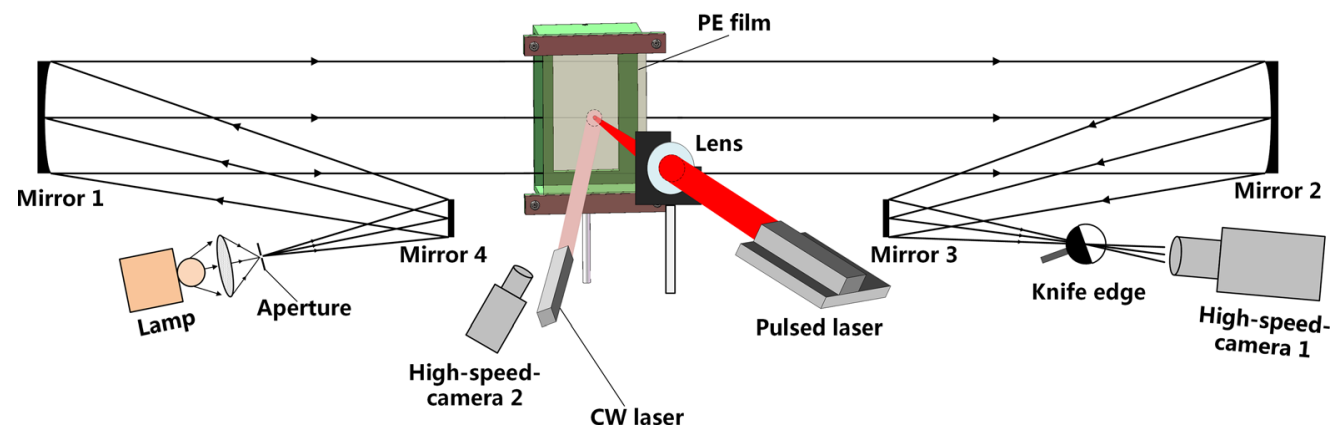

Fig. 1 Schematic of the test model. 


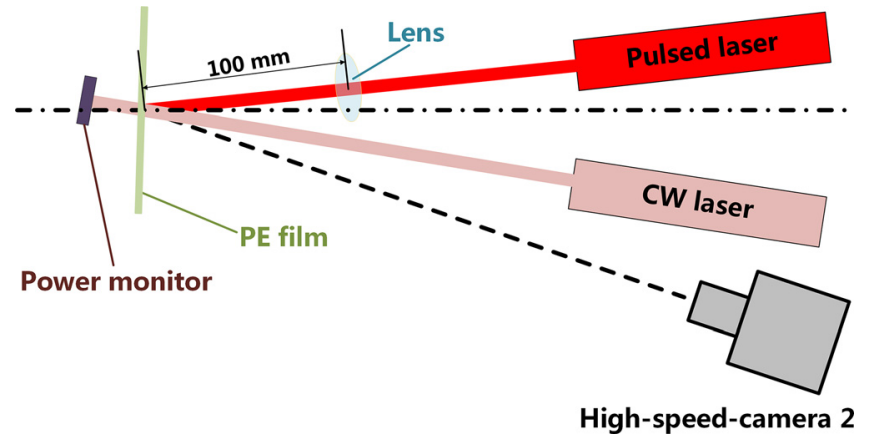

Fig. 2 Top view of the specific arrangement of the lasers in the tests.

The pulsed laser source was a flash-pumped solid-state yttrium aluminum garnet laser that delivered light pulses at a wavelength $\lambda$ of $1064 \mathrm{~nm}$, with a duration $\tau$ of 6 to $8 \mathrm{~ns}$ at a tunable repetition rate in the range of 0 to $5 \mathrm{~Hz}$ and pulse energy up to $70 \mathrm{~mJ}$. The pulsed laser could also be opened manually in single-pulse mode. The beam spot of the laser had a diameter of $4 \mathrm{~mm}$ and a beam divergence angle of $4 \mathrm{mrad}$. A lens with a focal length of $100 \mathrm{~mm}$ was used to concentrate the beam, and the PE film was located at the focal waist of the lens, as shown in Fig. 2. After focusing, the pulsed beam irradiated the PE film from close to the normal direction.

The CW laser source used was a CW fiber-optic laser with a beam wavelength of $1064 \mathrm{~nm}$. Before irradiation by the main beam, the laser produced a weaker beam that was used to align the laser. This is referred to as the beacon light of the laser. The $\mathrm{CW}$ beam was adjusted to irradiate the same location as the pulsed laser, as shown in Fig. 2. The diameter of the $\mathrm{CW}$ beam was $3 \mathrm{~mm}$, which was collimated before irradiating the PE film.

A Schlieren optics system was used for the flow field visualization of the side surface of the PE film when irradiated by the laser beam; the specific Schlieren arrangement is shown in Fig. 1. An iodide tungsten lamp acted as the source of illumination for the optics. Two spherical mirrors (mirrors 1 and 2) with a diameter of $100 \mathrm{~mm}$ and focal length of $2 \mathrm{~m}$ were located on the same main axis parallel to the surface of the PE film. Mirrors 3 and 4 were plane mirrors that were used to reflect the beam of the Schlieren optics. The aperture and knife edge were located at the focal waists of spherical mirrors 1 and 2, respectively. A high-speed camera (camera 1) with a frame frequency of 2000 or $125 \mathrm{~Hz}$ and an image resolution of $1024 \times 1024$ was used to record the flow field changes in the Schlieren optics. A Nikon lens with a fixed focal length of $200 \mathrm{~mm}$ was used with the high-speed camera.

In addition to the flow field visualization on the side surface of the PE film in the test, another high-speed camera (camera 2) with an image resolution of $1028 \times 1024$ and frame frequency $125 \mathrm{~Hz}$ was used to record the changes in the front surface, as shown in Fig. 1. The specific locations of the pulsed laser, CW laser, and high-speed camera 2 are shown in Fig. 2. The incident directions of the pulsed and CW lasers were nearly perpendicular to the PE film. AThorlabs optical power monitor was used to absorb the wasted power of the $\mathrm{CW}$ laser after the beam was transmitted through the PE film, and the recording frequency was $1 \mathrm{~Hz}$. A microscope was used offline after the tests to measure the surface damage caused to the PE film after irradiation with the pulsed laser.

\section{Results and Discussion}

\subsection{Effect of CW Laser Beam Alone}

When only a CW laser beam at a wavelength of 1064-nm irradiated the PE film, the PE film was not damaged by the beam. (Video 1, mp4, 6156 KB [URL: https://doi.org/10.1117/1.OE.59.12 .125103.1]. and Video 2, mp4, 3582 KB [URL: https://doi.org/10.1117/1.OE.59.12.125103.2].). Because of the transparent nature of the PE film, most of the laser energy passed through the film and the absorbed energy of the beam was insufficient to cause any damage to the surface of the film. When there was no film, the measured data were 0.92 and $5.23 \mathrm{~W}$, which reflected the power of the weak and strong CW laser, respectively. Based on the size of the light spot, 
the power densities of the weak and strong lasers were 13 and $74 \mathrm{~W} / \mathrm{cm}^{2}$, respectively. When the PE film of 0.6-mm thickness was positioned in the light path, the transmitted powers of the weak and strong lasers were 0.835 and $4.765 \mathrm{~W}$, respectively, which fell slightly. Ignoring the reflection from the surfaces of the PE film, the transmittances of the weak and strong lasers were evaluated to be $90.8 \%$ and $91.1 \%$, respectively. The transmitted power of the weak and strong laser remained constant after $\sim 10 \mathrm{~s}$, which showed that a $\mathrm{CW}$ laser alone with a wavelength of $1064 \mathrm{~nm}$ and the power of $5.23 \mathrm{~W}$ could not cut through the PE film. Further, a higher power of $70.4 \mathrm{~W}$ of the same CW laser was used to attempt to cut the PE film, but the PE film still could not be cut through. The corresponded power density was about $995 \mathrm{~W} / \mathrm{cm}^{2}$, which showed it was not easy for the $\mathrm{CW}$ laser of $1064 \mathrm{~nm}$ alone to cut through the PE film.

\subsection{Effect of Pulsed Laser Beam Alone}

When the CW laser was changed to a pulsed laser, the situation changed. Because the power level of the pulsed laser was low, its beam was focused by a lens before irradiating the PE film, as shown in Figs. 1 and 2. The diameter of the pulsed laser after focusing was evaluated as $d=4 \lambda f /(\pi D)$, where $\lambda$ is the laser wavelength, $f$ is the focal length of the lens, and $D$ is the diameter of the incident laser. The calculated diameter of the light spot after focus was $34 \mu \mathrm{m}$, and the power density of the laser beam at the focal waist was evaluated to be $\sim 1.1 \times$ $10^{12} \mathrm{~W} / \mathrm{cm}^{2}$. This power density was near to the threshold needed to induce the breakdown of the air, ${ }^{3}$ but there was no evident plasma produced after the lens when the pulsed laser was opened in the air.

Once the PE film was placed at the focal waist of the laser beam, a plasma was produced near the front surface of the film. Figure 3 shows the images of the flow field sequence of Schlieren optics after single laser pulse irradiation. The incident direction of the pulsed laser was along the negative $x$ axis direction of the figure. The high-speed camera 1 was opened with a frame frequency of $2000 \mathrm{~Hz}$, and the pulsed laser was opened manually in the recording process of the high-speed camera. The time $0 \mathrm{~ms}$ in Fig. 3 corresponded to the time when the recorded images
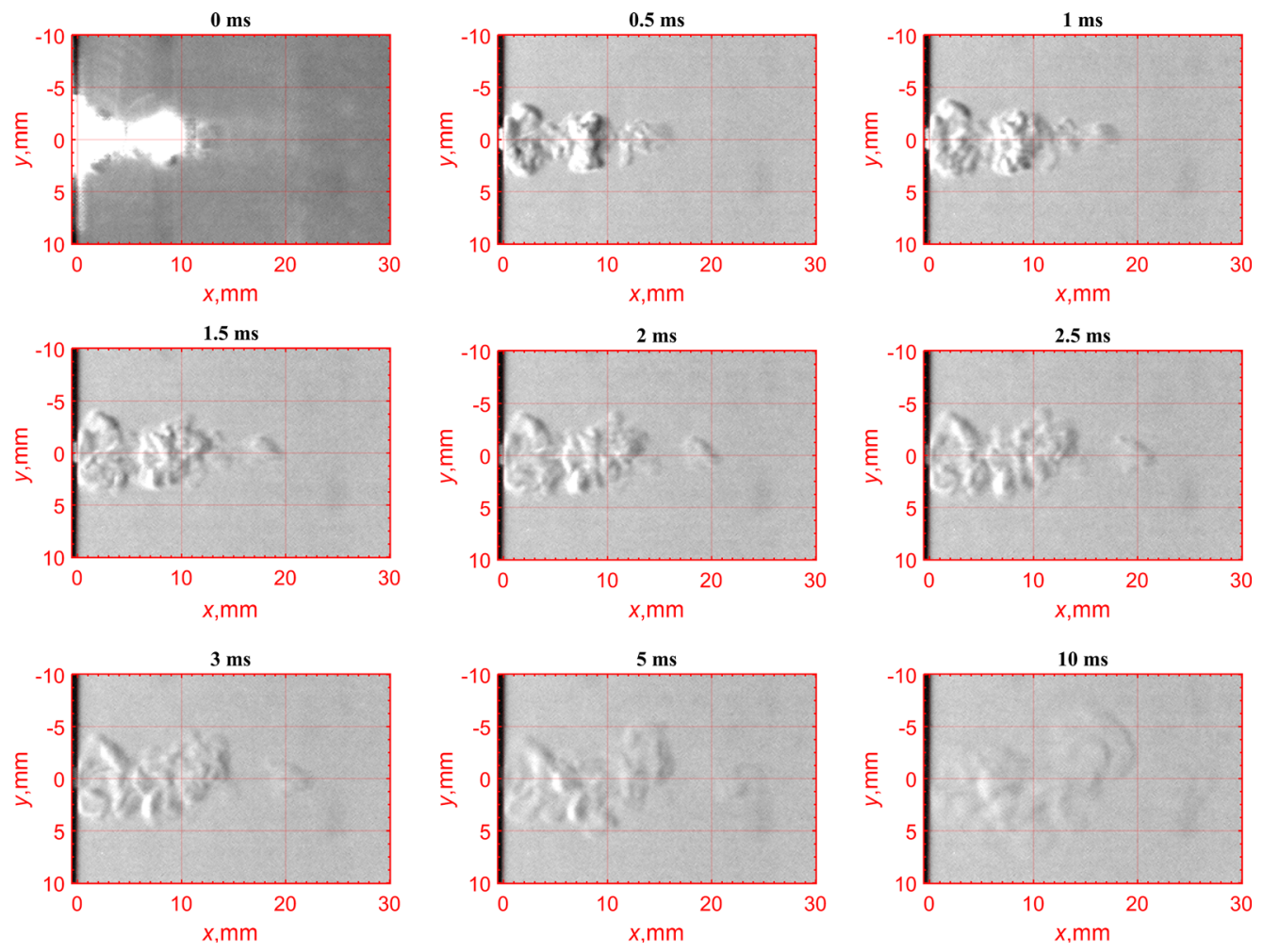

Fig. 3 Flow field sequences of PE film after a single laser pulse. 
first captured the very bright signal of the laser-induced plasma, and the recorded image sequences were spaced 0.5-ms apart for the duration of the test.

Based on the laser-induced plasma research of Brieschenk et al., ${ }^{6}$ the time scales for the plasma emission lifetime were of the order of tens of microsecond. The exposure time of high-speed camera 1 was set to $499.75 \mu$ s; thus, the Schlieren imaging system could not record the complex forming process of the plasma. Based on the ablation of opaque materials, ${ }^{11}$ the film undergoes complex processes such as surface absorption, material excitation, temperature rise, thermal vaporization, nonthermal ablation, plasma formation, particle ejection, and gas plume. The evolution of the post-plasma gas plume was recorded in Fig. 3, and it developed over a time scale duration of $\sim 10 \mathrm{~ms}$. A jet similar to that in the laser-induced breakdown process was recorded at $0 \mathrm{~ms}$, which propagated toward the laser source. The moving velocity of the main structures of the jet was evaluated to be $<10 \mathrm{~m} / \mathrm{s}$ after $0 \mathrm{~ms}$, as shown in Fig. 3. In addition, the image shapes of the formed jets for different test cases were different when the irradiated positions were changed from one clean place to another on the PE film, which showed random shapes of the jets.

The damage characteristics of the PE film are shown by the enlarged images from the microscope. After the PE film was irradiated by the pulsed laser, the damaged part was cut down and placed on the stage of the microscope to be photographed offline. The visible surface change of the PE film corresponding to the irradiation of the same single laser in Fig. 3 is shown in Fig. 4. A small circular crater with a radius of $\sim 0.15 \mathrm{~mm}$ was evident in the PE film. The spots around the hole were the ablated particles ejected from the crater, which showed that the plasma was sprayed out from the surface of the PE film. In addition, because the PE film was ablated under ambient conditions, the ablated material was rapidly carbonized in the air. The opaque black parts in and around the crater were carbonized PE materials after cooling. The craters on the PE film differed slightly for each different case in the same way as the shape of the jet varied.

Figures 3 and 4 show the effect of single laser pulse irradiating at different positions on a PE film. We also need to understand the effect when multiple laser pulses irradiate the same position with the film and laser source. Because the flow field images changed at different times after the opening of the pulsed laser as shown in Fig. 3, we needed to open the pulsed laser with the same time intervals as before to compare the flow field. The flow field images corresponding to the time interval of $0.5 \mathrm{~ms}$ were compared. The flow field changes when different numbers of laser pulses irradiated the same position on the film. The shapes of the ejected gas plumes for the different numbers of laser pulses varied. This shows the inconsistency of the jet shapes based on the time resolution of the Schlieren system. Because each laser pulse was opened by hand in the test, the time resolution of the Schlieren system and the exposure time of the high-speed camera $1(599 \mu \mathrm{s})$ were both much higher than the time scale of the laser-induced plasma $(10 \mu \mathrm{s})$. The exact time of each laser pulse after the opening of the laser may not have been exactly the same. Also, based on the current Schlieren images, the ejected plume showed random

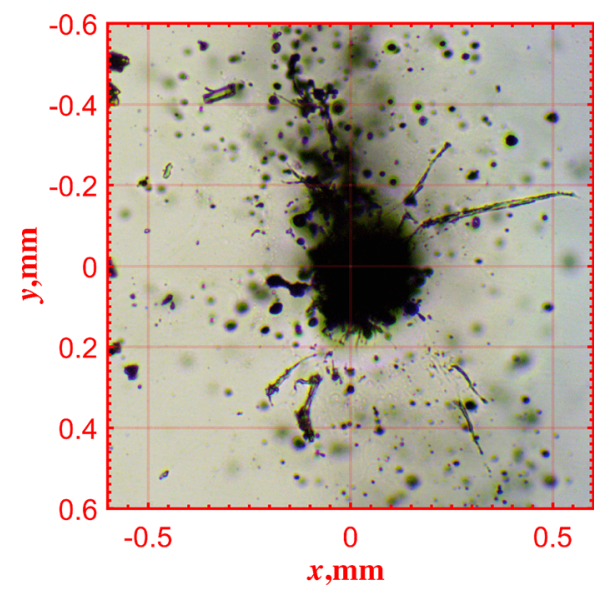

Fig. 4 Surface damage of PE film after a single laser pulse. 

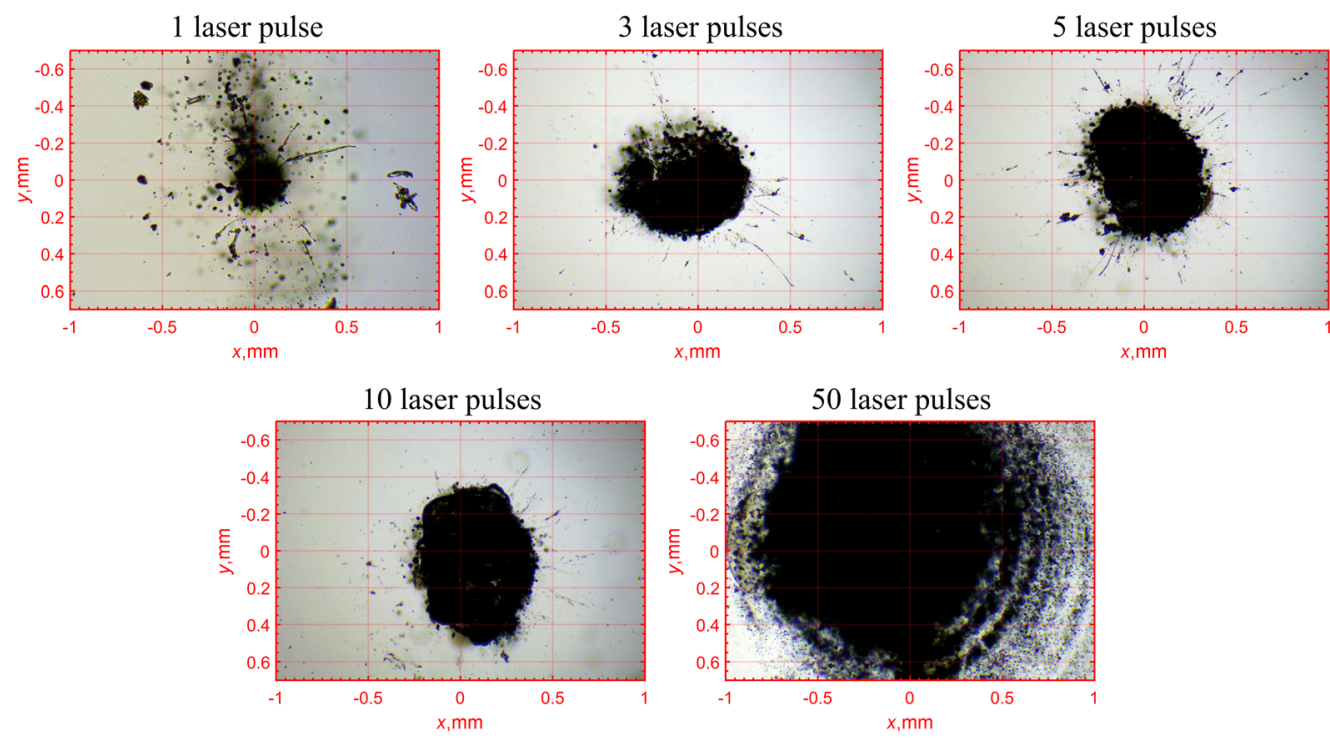

Fig. 5 Microscope images of surface changes of the PE film after being irradiated by different numbers of laser pulses.

shapes at different positions of the film, so there was no evident consistency in the changes of the plume shapes.

Although the ejected gas plume shape images from a series of laser pulses varied, the surface changes were more obvious as shown in the magnified microscope pictures in Fig. 5, and the images photographed with the front camera. When more laser pulses irradiated the same position on the PE film, the initial carbonized parts absorbed more energy from the incident laser beam. More ablated particles were ejected through the surface. The crater on the surface became larger and more ablated particles were deposited around the crater. There was a positive relationship between the number of laser pulses and the damaged area on the surface of the PE film. As more laser pulses irradiated the film, so the expansion rate of the damaged parts on the film increased.

Although the damaged area on the front surface of the PE film got worse as the number of laser pulses increased, the lasers still could not easily cut through the PE film.

\subsection{Effect of Successive Pulsed and CW Laser Beams}

The CW laser of 1064-nm wavelength could not damage the surface of the transparent PE film owing to the low absorption of the laser energy; however, the pulsed laser after focusing did change the surface state of the PE film. It would therefore be of interest to investigate the effect of the CW laser on a film with ablation damaged caused by laser pulses.

The CW laser transmittance of the PE film after irradiation with different numbers of laser pulses needed to be measured first. The evaluated weak and strong CW laser transmittances of the PE film were almost equal to each other. The weak CW laser would have an insignificant effect on the surface of the PE film damaged by the pulsed laser; therefore, the transmittances of the damaged PE film were evaluated using the weak $\mathrm{CW}$ laser. Ignoring the reflection, the recorded data of the power monitor were used to calculate the transmittance of the film as above, and the results are shown in Fig. 6.

When one laser pulse was used, the transmittance remained almost the same as with no pulsed laser, and the decrease in transmittance rates was small when the number of laser pulses increased from one to three. The decrease was significantly more rapid for more than three laser pulses. As described above, as the numbers of the laser pulses irradiating the film increased, more carbonized particles ejected from the crater were deposited near the crater, and the crater became larger on the PE film surface. The carbonized part on the PE film was opaque with a lower transmittance that decreased as more laser pulses were used.

After the PE film was damaged by a certain number of laser pulses, a strong CW laser was used to irradiate the PE film in the same position. The pulsed laser was focused; however, the 


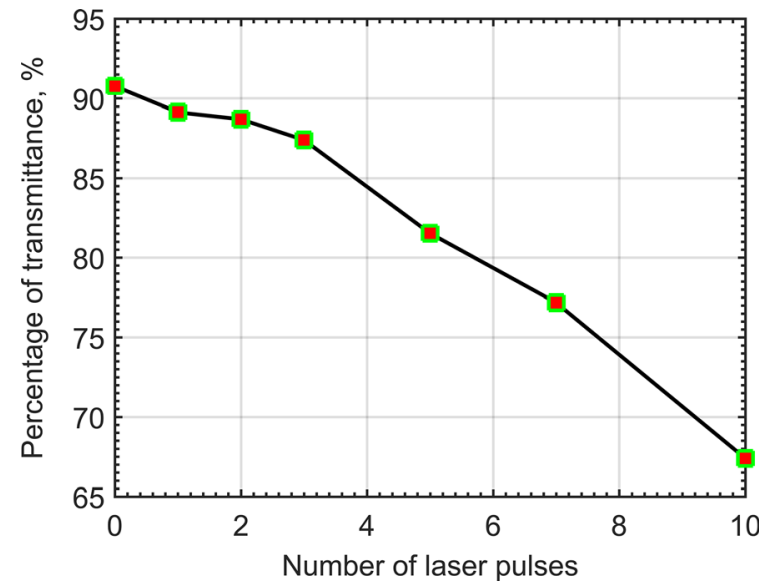

Fig. 6 Transmittance of PE film after being irradiated by different numbers of laser pulses.

CW laser was not concentrated, and the light spot of the CW on the surface (diameter $3 \mathrm{~mm}$ ) was much larger than that of the pulsed laser (with diameter $34 \mu \mathrm{m}$ ). The surface modification due to the pulsed laser therefore only affected a very small portion of the PE area illuminated by the CW laser. When the $\mathrm{CW}$ laser began to irradiate, a power monitor was used to measure the transmitted energy through the PE film, and the Schlieren optics and the front high-speed camera were used to record the changes in the PE film from both the side and front surfaces simultaneously. The frame frequencies of the high-speed cameras were set to $125 \mathrm{~Hz}$, and the exposure time was $1 / 126 \mathrm{~s}$.

The measured power profiles of the $\mathrm{CW}$ laser after transmission through the PE film are shown in Fig. 7, where the time $0 \mathrm{~s}$ in the $x$ axis corresponded to the moment when the strong $\mathrm{CW}$ laser was opened. When the $\mathrm{CW}$ laser began to irradiate the film after irradiation by a single laser pulse, the initial carbonized PE materials absorbed some of the energy of the CW laser, causing more PE materials to be carbonized and decreasing the transmitted power. A short time later, this carbonizing process stopped. The total area of the carbonized region on the film was so small relative to the area of the beam that the carbonizing effect remained low. The power of the transmitted laser tended to be stable after initially decreasing a little. The stable transmittance of the damaged PE film on the $\mathrm{CW}$ laser was evaluated to be $76 \%$. The recorded images from the side and front surfaces are shown in Fig. 8. No matter how long the $\mathrm{CW}$ laser was irradiating the surface, the state of the PE film remained stable, and the images from both high-speed cameras were unchanged as shown in Fig. 8. We can conclude, therefore, that the current $\mathrm{CW}$ laser was unable to cut through the PE film with damage caused by only one laser pulse.

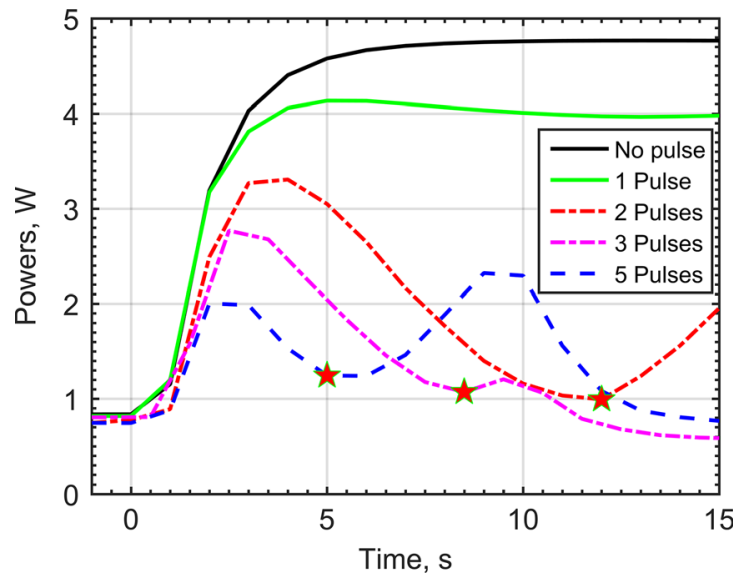

Fig. 7 Power profiles of CW laser through the PE film after irradiation by different numbers of laser pulses ( $0 \mathrm{~s}$ corresponded to the time the strong $\mathrm{CW}$ laser was opened, and stars corresponded to the times when the PE film was cut through). 


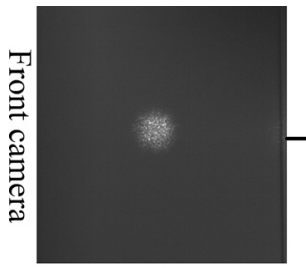

$0 \mathrm{~s}$

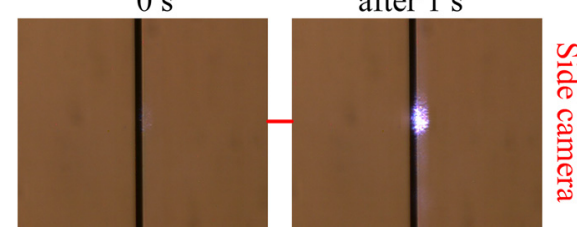

Fig. 8 Visualized images during the test with one laser pulse irradiated

When the PE film was first irradiated by two laser pulses, the scenario changed, as shown in Fig. 7. The transmitted power stopped increasing sooner than that with a single laser pulse, and then it decreased gradually. At $12 \mathrm{~s}$, the received power of the monitor started increasing again over time. The images of the PE film during this process are shown in Fig. 9. When the CW laser began to irradiate the PE film, the initial carbonized parts absorbed the $\mathrm{CW}$ laser energy, and a larger area of the PE film was carbonized. With more carbonized regions in the film, more CW laser energy was absorbed, and the carbonizing process accelerated with a decrease in transmitted power. In contrast to the test of one laser pulse, this continuous carbonization process did not stop but continued until the irradiated part of the PE film within the range of the light spot of the CW laser was burned.

In the test, the PE film began to smoke gradually, as seen in the images from the front and side high-speed cameras, and more smoke appeared over time. Because the smoke scattered the CW laser in all directions, the bright light spot in the front and side cameras indicated the amount of

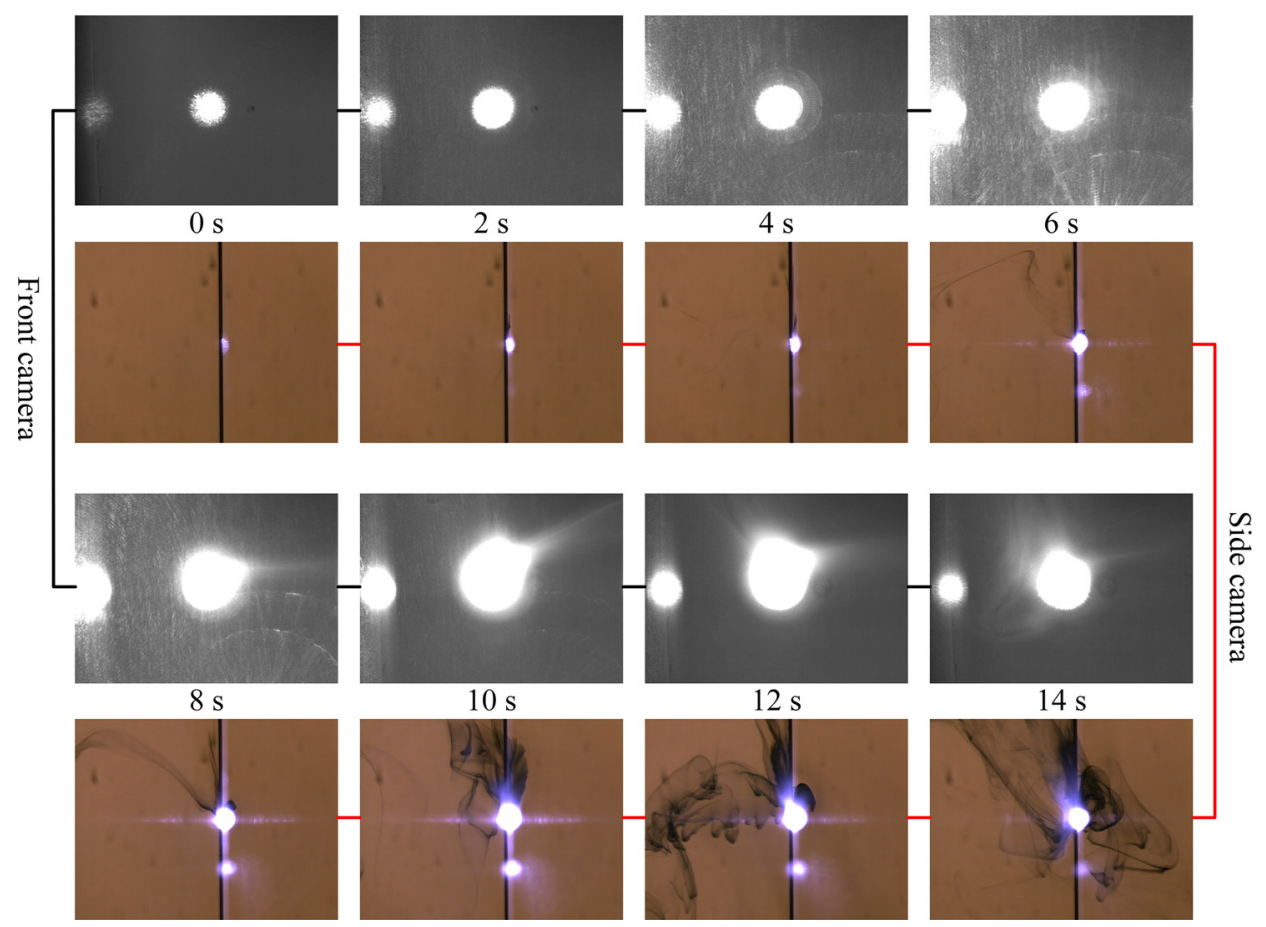

Fig. 9 Image sequences during the test with two laser pulses irradiated (Video 3, mp4, $10343 \mathrm{~KB}$ [URL: https://doi.org/10.1117/1.OE.59.12.125103.3]. and Video 4, mp4, 10929 KB [URL: https:// doi.org/10.1117/1.OE.59.12.125103.4]. ). 
Table 1 Times to cut through PE film after being irradiated by different numbers of laser pulses.

\begin{tabular}{lccccc}
\hline \hline Numbers of the laser pulses & 0 & 1 & 2 & 3 & 5 \\
\hline Time, s & $\infty$ & $\infty$ & 12 & 8 & 5.5 \\
\hline \hline
\end{tabular}

smoke generated. The light spot in the front and side cameras first increased over time, and then decreased gradually. The carbonized materials were turned into smoke by the $\mathrm{CW}$ laser, with more smoke near the surface of the PE film. Once the irradiated part of the PE film was cut through by the $\mathrm{CW}$ laser, the received power began to increase over time, and less smoke was produced. The turning point in the curve of the received power, which stopped decreasing over time, can be seen as the moment when the PE film was cut through. We can see that the time to cut through the PE film was $12 \mathrm{~s}$ after it was first damaged by two laser pulses.

When the PE film was irradiated by more laser pulses, the images from the front and side cameras were similar to the results of Fig. 9. In the test, once the visualized smoke decreased over time, the CW laser was closed manually for the PE film had been cut through. The CW laser was closed at the time of $9 \mathrm{~s}$ when three laser pulses were irradiated, and at $9.5 \mathrm{~s}$ for the curve when five laser pulses were irradiated, which caused a decrease in the curves of the received powers of the $\mathrm{CW}$ laser in Fig. 7. Based on the analysis of the test cases with 1 and 2 laser pulses irradiated, the specific times taken to cut through the PE film, after irradiation by different numbers of laser pulses, are given in Table 1. The specific cut-through times were also marked with stars in Fig. 7.

When more laser pulses irradiated the PE film first, the cut through time was shorter. The results here can help the near-infrared laser cut transparent materials that have low absorption, such as PE film, and greatly reduce the threshold for the $\mathrm{CW}$ laser to cut through the material.

\section{Conclusions}

The PE film was irradiated by a CW laser alone, a pulsed laser alone, and then by different numbers of laser pulses followed by a $\mathrm{CW}$ laser. The irradiating processes were recorded by a Schlieren optics system on the side surface of the film and by a high-speed camera on the front surface. The transmitted energy of the $\mathrm{CW}$ laser was measured using a power monitor.

The results show that it is difficult for a CW laser with a wavelength of $1064 \mathrm{~nm}$ to cut through a transparent material such as PE film due to the low level of absorption of the laser energy. When the PE film was placed at the focal waist of a pulsed laser beam, a plasma was induced near the surface of the film, and a gas jet ejected from the surface of the film, which propagated towards the direction of the laser source. After irradiation by the pulsed laser, a small crater formed on the surface of the film. This crater and the deposited carbonized materials decreased the transmittance of the film. With more laser pulses irradiation of the film, the $\mathrm{CW}$ laser transmittance of the PE film decreased further. Once the PE film was damaged by more than two laser pulses at the same position, the threshold for the CW laser to cut through the film was greatly decreased.

\section{Acknowledgments}

The authors would like to thank Yan Zhou, Mi Yang, and Shiwen Shu for their help in the experiments.

\section{References}

1. R. Moser et al., "Laser processing of gallium nitride-based light-emitting diodes with ultraviolet picosecond laser pulses," Opt. Eng. 51(11), 114301 (2012).

2. H. Chen et al., "Laser plasma-induced damage characteristics of $\mathrm{Ta}_{2} \mathrm{O}_{5}$ films," Opt. Mater. Express 9(7), 3132-3145 (2019). 
3. P. Chylek et al., "Pressure dependence of the laser-induced breakdown thresholds of gases and droplets," Appl. Opt. 29(15), 2303-2306 (1990).

4. T. X. Phuoc, "Laser-induced spark ignition fundamental and applications," Opt. Lasers Eng. 44, 351-397 (2006).

5. G. Sinibaldi et al., "Laser induced cavitation: plasma generation and breakdown shockwave," Phys. Fluids 31, 103302 (2019).

6. S. Brieschenk, S. O'byrne, and H. Kleine, "Visualization of jet development in laserinduced plasmas," Opt. Lett. 38(5), 664-666 (2013).

7. S. Brieschenk et al., "High-speed time-resolved visualisation of laser-induced plasma explosions," Proc. SPIE 7126, 71260N (2020).

8. Y. Ding, L. Yang, and M. Hong, "Enhancement of pulsed laser ablation assisted with continuous wave laser irradiation," Sci. China, Phys. Mech. Astron. 62(3), 034211 (2019).

9. M. Zhou et al., " $\mathrm{TiO}_{2} / \mathrm{SiO}_{2}$ dielectric film damage induced by combination of pulsed and continuous wave infrared laser irradiation," in Third Int. Symp. Laser Interaction with Matter, Jiangsu (2014).

10. I.-B. Sohn et al., "Laser ablation of polypropylene films using nanosecond, picosecond, and femtosecond laser," J. Opt. Soc. Korea 12(1), 38-41 (2008).

11. B. Rethfeld et al., "Timescales in the response of materials to femtosecond laser excitation," Appl. Phys. A Mater. Sci. Process. 79, 767-769 (2004).

Changqing Song is a research assistant at the College of Advanced Interdisciplinary Studies, working in the State Key Laboratory of Pulsed Power Laser Technology, National University of Defense Technology, Changsha, China.

Kai Han is a research associate at the College of Advanced Interdisciplinary Studies, working in the State Key Laboratory of Pulsed Power Laser Technology, National University of Defense Technology.

Xiao Li is a research associate at the College of Advanced Interdisciplinary Studies, working in the State Key Laboratory of Pulsed Power Laser Technology, National University of Defense Technology.

Wenda Cui is a research assistant at the College of Advanced Interdisciplinary Studies, working in the State Key Laboratory of Pulsed Power Laser Technology, National University of Defense Technology.

Xiaojun Xu is a professor at the College of Advanced Interdisciplinary Studies, working in Hunan Provincial Key Laboratory of High Energy Laser Technology, National University of Defense Technology. 